\title{
Performance motora de lactentes prematuros segundo a Alberta Infant Motor Scale:
}

\section{Uma revisão de literatura}

\author{
Motor performance of premature infants according to Alberta Infant Motor Scale: A literature \\ review
}

Desempeño motor de lactantes prematuros según la Escala Motor Infantil Alberta: Revisión de la

literatura

Recebido: 22/05/2021 | Revisado: 29/05/2021 | Aceito: 09/06/2021 | Publicado: 21/06/2021

\author{
Gabriela Menezes da Silva \\ ORCID: https://orcid.org/0000-0001-6202-0144 \\ Faculdade Independente do Nordeste, Brasil \\ E-mail: menezesgabriela98@gmail.com \\ Afonso José Mafra Freire \\ ORCID: https://orcid.org/0000-0002-5088-6255 \\ Faculdade Independente do Nordeste, Brasil \\ E-mail: affonsofreire9@gmail.com \\ Laisla Pires Dutra \\ ORCID: https://orcid.org/0000-0003-4111-7101 \\ Faculdade Independente do Nordeste, Brasil \\ E-mail: laysla19@hotmail.com
}

\begin{abstract}
Resumo
Introdução: Nos últimos anos um número expressivo de crianças prematuras vem nascendo no Brasil, associado a este dado estão as condições de risco; morbimortalidade neonatal ocorrendo antes do primeiro ano de vida. A importância em se pensar na assistência à saúde da criança prematura, durante os primeiros anos de vida é essencial, afim de minimizar riscos sociais, biológicos e consequente riscos para desenvolvimento infantil no qual o lactente prematuro está exposto, por isso a importância de mensurar o desenvolvimento motor de prematuros respeitando a idade gestacional corrigida. Analisar os estudos que utilizaram o instrumento de avaliação de triagem do desenvolvimento motor Alberta Infant Motor Scale (AIMS) em lactentes prematuros. Metodologia: Trata-se de um artigo do tipo revisão de literatura, no qual foram utilizadas as seguintes bases de dados: PubMed, SciELO, LILACS e Google Acadêmico, selecionando artigos publicados entre 2010-2021. Foram selecionados para construção do atual estudo 16 artigos, destes 10 foram escolhidos para construção do atual estudo, no qual emergiram dois eixos de discussão: As baixas condições socioeconômicas e atraso no desenvolvimento motor; e o tempo prolongado de internamento na UTIN e o desempenho motor. Conclusão: Os resultados evidenciaram que a Alberta Infant Motor Scale (AIMS)é considerado um instrumento de triagem do desenvolvimento motor grosso confiável para ser aplicados em bebês prematuros, sendo evidenciado também que as condições socioeconômicas bem como o tempo de internamento na UTIN são fatores de risco para o desenvolvimento motor infantil.
\end{abstract}

Palavras-chave: Prematuridade; Desenvolvimento infantil; Saúde da criança.

\begin{abstract}
Introduction: in the last few years, a significant number of premature children have been born in Brazil, associated with this information are the risk conditions of neonatal morbi mortality wich is occurring during the first year of life. The importance of the thought about medical assistance for the premature child during its very first years is essential, and have the purpose of minimizing the social and biological risks and the consequent child development to which the infant is exposed, this shows the relevance in the measuring the motor development of the premature child, with respect to the corrected gestation age. The analysis of previous paper published that used as instrument of evaluation of triage infant motor development Alberta Motor Scale (AIMS) for premature infants. Methodology: It is an article of the type of literature research, whose source comes from: PubMed, SciELO, LILACS and academic Google, there were selected sixteen published papers from the 2010-2021. Sixteen articles were selected for the construction of the current study, of these, ten were chosen for the construction of the current study, in which two axes of discussion emerged: Low socioeconomic conditions and delayed motor development; and the prolonged length of stay in the NICU and motor performance. Conclusion: The results have shown that the Alberta infant Motor Scale (AIMS) is considered as an screening instrument of the gross motor development with reliability in its application to premature babies, also is an evidence of the socioeconomic conditions, as well as the time spent in the UTIN are risk factors for the child motor development.
\end{abstract}


Keywords: Prematurity; Child development; Children health.

\section{Resumen}

Introducción: Los últimos años un número significativo de niños prematuros ha nacido en Brasil, asociados a este dato están las condiciones de riesgo; Morbimortalidad neonatal antes del primer año de vida. La importancia de pensar en la asistencia a la salud del niño prematuro, durante los primeros años de vida, es fundamental, con el fin de minimizar los riesgos sociales, biológicos y los consiguientes riesgos para el desarrollo infantil al cual el lactante prematuro está expuesto, por lo que la importancia de medir el desarrollo motor de prematuros respetando la edad gestacional corregida. Analizar los estudios que utilizaron la herramienta de evaluación de selección del desarrollo motor de Alberta Infant Motor Scale (AIMS) en lactentes prematuros. Metodología: Se trata de un artículo de revisión de la literatura, en el que fueron utilizadas las siguientes bases de datos: PubMed, SciELO, LILACS y Google Académico, seleccionando artículos publicados entre 2010-2021. Fueron usados 16 artículos para la construcción del presente estudio. De esos 10 fueron elegidos para construir el presente estudio, en el que surgieron dos ejes de discusión: las bajas condiciones socioeconómicas y desarrollo motor retardado; y la estancia prolongada en la UTIN y el desempeño motor. Conclusión: Los resultados mostraron que Alberta Infant Motor Scale (AIMS) es considerado una herramienta de selección del desarrollo motor grueso confiable que se aplica a los bebés prematuros, y también se muestra que las condiciones socioeconómicas, así como la duración de la estadía en la UTIN son factores de riesgo para el desarrollo motor del niño.

Palabras clave: Prematuridad; Desarrollo infantil; Salud del niño.

\section{Introdução}

A organização mundial de saúde (OMS) define prematuro, todo bebê cujo nascimento ocorreu antes de 37 semanas de idade gestacional. O baixo peso ao nascer é caracterizado por neonatos que nascem com peso inferior a $2500 \mathrm{~g}$, juntamente com a prematuridade são condições preditivas de risco para a morbidade e mortalidade neonatal. Cerca de 20 milhões de crianças prematuras ou com baixo peso nascem anualmente, o Brasil apresenta um terço destes nascimentos correspondendo a mortalidade infantil antes do primeiro ano de vida (OMS, 2018; Sampaio, et al., 2015; Carvalho, et al., 2016).

O Brasil não é bem representado quando o assunto em pauta de discussão é prematuridade, sendo citado em relatório da organização mundial de saúde como as 10 nações com maiores taxas de nascimento prematuro, equivalente a países como Estados Unidos, Índia e Nigéria, o que revela um problema mundial. Os resultados gestacionais negativos são influenciados por fatores que se comunicam entre si, como os fatores biológicos, sociais e ambientais, aos quais a gestante está exposta durante o período gestacional. Os partos prematuros também se relacionam em torno de 40 a $50 \%$ a causas desconhecidas e idiopáticas (Balbi \& Carvalhaes \& Parada, 2015; Carvalho, et al., 2016).

Os resultados negativos advindos do parto prematuro estão diretamente correlacionados a idade gestacional, o que significa que o amadurecimento bem como um número menor de complicações neonatais, variam de acordo com a idade gestacional; ou seja, cada semana do feto intrautero marca um maior amadurecimento dos sistemas. Entretanto quanto menor o peso e a idade gestacional do bebê prematuro, maior a probabilidade de eventos biológicos ocorrer, como: hemorragia periintraventricular, displasia broncopulmonar, leucomalácia, maior exposição em suporte de ventilação mecânica e retinopatia da prematuridade, aumentando ainda o risco para desenvolvimento típico (Vanin, et al., 2020; Fuentefria \& Silveira \& Procianoy, 2017).

A prematuridade é considerada fator de risco para o desenvolvimento infantil, as condições biológicas e sociais apresentadas durante os períodos, pré, peri e pós-natal, contribuem para maiores chances de déficits motores, o que implica em atraso no desenvolvimento da criança. O desenvolvimento como um todo corresponde a comunicação entre os sistemas muscular, nervoso, para com o ambiente. O primeiro ano de vida do lactente prematuro é essencial para o seu desenvolvimento, pois é o período no qual observa-se maior número de transformações, é também o período em que o SNC dessa criança se encontra em processo de formação, corresponde ainda ao momento em que as habilidades relacionadas ao movimento são aprendidas com mais rapidez. (Sá, et al., 2017; Alves \& Campos, 2011). 
O cuidado à saúde do recém-nascido prematuro provoca redução dos riscos sociais e biológicos, o que leva a contribuições no decorrer da vida. A intervenção precoce corresponde ao acompanhamento desde o nascimento até os três anos de vida, proporcionando um conjunto de programas multidisciplinares que visam a prevenção e promoção da saúde da criança. É justamente durante os primeiros anos que a intervenção precoce deve ocorrer, nesta fase as capacidades motoras devem ser avaliadas por meio de programas de acompanhamento ao prematuro, como o follow-up, de forma a avaliar o desenvolvimento ao longo do primeiro ano de vida do recém nato, intervindo precocemente, com o objetivo de reduzir alterações do nascimento imaturo (Ferreira, et al., 2020; Silva, et al., 2011).

Em se tratando especificamente do processo de avaliação do desempenho motor a escala de Albert Motor Infant Scale (AIMS) está entre os instrumentos de avaliação mais utilizados para descrever o comportamento motor e as habilidades motoras realizadas por lactentes prematuros, permitindo ainda avaliar a qualidade do movimento, sendo esta escala validada para população infantil brasileira (Fuentefria \& Silveira \& Procianoy, et al., 2017).

A Albert Infant Motor Scale (AIMS) de avaliação leva em conta não somente a definição de desenvolvimento motor, mas também considera o desenvolvimento motor de acordo com a maturação do SNC, sua interação com o meio, e organização do processo de desenvolvimento. Trata-se de uma escala de caráter observacional do desempenho motor da criança (Mello, et al., 2014).

Diante desse contexto, o presente estudo tem como objetivo analisar os estudos que utilizaram o instrumento de avaliação de triagem do desenvolvimento motor Alberta Infant Motor Scale (AIMS) em lactentes prematuros.

\section{Metodologia}

Trata-se de um estudo de revisão integrativa de literatura de abordagem qualitativo, caracterizado pela análise de pesquisas cujo embasamento teórico permite síntese acerca das considerações elucidadas ao longo dos estudos. A construção desta revisão se deu por meio de definição dos descritores, busca pela base de dados, critérios de inclusão e exclusão, seleção dos estudos e avaliação do conteúdo sobre o tema em questão. Um estudo qualitativo é aquele que torna importante a interpretação do pesquisador com suas posições sobre o fenômeno em estudo. A coleta de dados geralmente acontece através de entrevistas com questões. A pesquisa qualitativa, usualmente, é realizada em meio natural com coleta direta de dados e o observador é o principal instrumento. (Pereira, et al., 2018)

Para a seleção dos artigos foram consultadas as seguintes bases de dados: National Library of Medicine (PubMed), Scientific Electronic Library Online (SciELO), Literatura Latino-Americana e do Caribe em Ciências da Saúde (Lilacs) e Google Acadêmico. A busca foi realizada através dos descritores: Prematuridade, Desenvolvimento Infantil e Saúde da criança.Foram estabelecidos como critérios de inclusão: artigos publicados entre 2010-2021, que tiveram análise quantitativa, que apresentaram o tema proposto pelo estudo em questão, com texto disponível na íntegra. Foram excluídos artigos que não apresentaram data de publicação correspondente a mais de 10 anos, artigos com análise quantitativa insuficiente para uma análise estatística confiável. Foram encontrados 16 artigos, destes, 10 foram incluídos para construção do atual estudo.

\section{Resultados}

Compuseram a amostra deste estudo 10 artigos que atenderam aos critérios de inclusão propostos, conforme apresentado no Quadro 1, segundo autor/ano, tipo de estudo, população e resultados. 
Quadro 1. Artigos selecionados para o desenvolvimento do estudo.

\begin{tabular}{|c|c|c|c|}
\hline AUTOR/ANO & $\begin{array}{l}\text { TIPO DE } \\
\text { ESTUDO }\end{array}$ & POPULAÇÃO & RESULTADOS \\
\hline $\begin{array}{l}\text { Formiga, Cezar \& } \\
\text { Linhares, } 2010\end{array}$ & $\begin{array}{l}\text { O estudo foi } \\
\text { prospectivo }\end{array}$ & $\begin{array}{l}10 \text { crianças pré-termo com baixo } \\
\text { peso ao nascer, de ambos os sexos } \\
\text { (sendo cinco meninos e cinco } \\
\text { meninas), avaliadas no Hospital } \\
\text { Materno Infantil (HMI) de } \\
\text { Goiânia. Todas as crianças } \\
\text { tiveram suas idades corrigidas } \\
\text { para o termo ( } 40 \text { semanas, como } \\
\text { preconizado pela Organização } \\
\text { Mundial de saúde. }\end{array}$ & 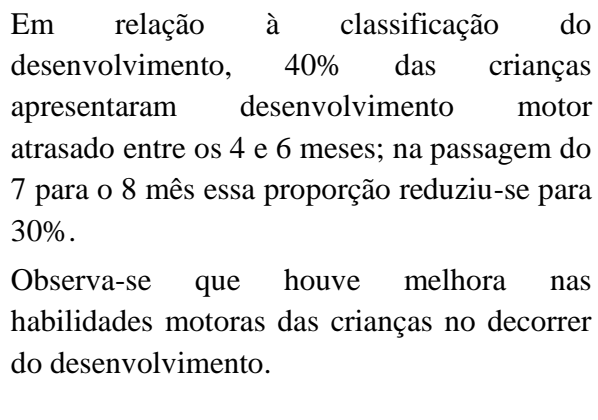 \\
\hline Giachetta, et al; 2010 & $\begin{array}{l}\text { Estudo do tipo } \\
\text { transversal }\end{array}$ & $\begin{array}{l}\text { Foram estudados } 67 \quad \mathrm{RN} \text {, } \\
\text { distribuídos em dois grupos } \\
\text { segundo o tempo de } \\
\text { hospitalização. Ao final da coleta } \\
\text { de dados, foi calculada a mediana } \\
\text { do tempo total de internação e os } \\
\text { grupos foram: grupo A, formado } \\
\text { por RN (n-35) com período de } \\
\text { internação menor ou igual a } 34 \\
\text { dias; e grupo B, formado por RN } \\
\text { (n-32) com período de } \\
\text { hospitalização maior que } 34 \text { dias. }\end{array}$ & $\begin{array}{l}\text { No presente estudo, os RN que apresentaram } \\
\text { escores compatíveis com atraso do } \\
\text { desenvolvimento motor, segundo a AIMS, } \\
\text { foram os que permaneceram mais tempo } \\
\text { (média de } 50 \text { dias) internados (grupo B) do } \\
\text { que os demais (grupo A), que apresentaram } \\
\text { escores compatíveis com o desenvolvimento } \\
\text { esperado para a idade gestacional corrigida, } \\
\text { não apresentando déficits no desenvolvimento } \\
\text { motor. }\end{array}$ \\
\hline Ribeiro, et al., 2010 & $\begin{array}{l}\text { Prospectivo } \\
\text { longitudinal }\end{array}$ & $\begin{array}{l}\text { Constituído por uma amostra de } \\
12 \text { bebês prematuros, de ambos os } \\
\text { sexos. } \\
\text { Foram incluídos os bebês com IG } \\
\text { < } 37 \text { semanas completas, peso ao } \\
\text { nascer } \leq 2500 \text { g, e frequência de } \\
\text { no mínimo } 1 \text { x/mês no PIP do } \\
\text { ambulatório de Fisioterapia do } \\
\text { Hospital Regional de Taguatinga- } \\
\text { Distrito Federal (HRT-DF). A } \\
\text { intervenção ocorreu no período de } \\
8 \text { meses, e houve média de } 12 \\
\text { sessões por bebê no programa de } \\
\text { intervenção precoce. }\end{array}$ & $\begin{array}{l}\text { Quanto ao DM amplo dos bebês pela AIMS, } \\
\text { observou-se que, aos } 4 \text { e } 5 \text { meses de IC, todos } \\
\text { os bebês foram classificados como típico e } \\
\text { suspeito. Porém, a partir do } 6^{\circ} \text { mês de IC, um } \\
\text { bebê apenas ( } 8 \% \text { ) apresentou DM amplo } \\
\text { classificado como atípico, o qual perdurou até } \\
\text { a última avaliação. Em relação aos padrões de } \\
\text { referência da AIMS, os prematuros estudados } \\
\text { apresentaram atraso significativo no DM } \\
\text { amplo nos três primeiros meses de avaliação. }\end{array}$ \\
\hline $\begin{array}{l}\text { Sartori, Saccani \& } \\
\text { Valentini, } 2010\end{array}$ & $\begin{array}{l}\text { Descritivo, de } \\
\text { abordagem } \\
\text { transversal }\end{array}$ & $\begin{array}{l}\text { A amostra foi dividida em dois } \\
\text { grupos: o grupo A, composto por } \\
40 \text { bebês de mães adolescentes } \\
\text { ( } 12 \text { a } 19 \text { anos), e o grupo B, } \\
\text { composto por } 40 \text { bebês com idade } \\
\text { e sexo pareados ao grupo A, de } \\
\text { mães adultas (acima de } 20 \text { anos). } \\
\text { Foram incluídos todos os bebês } \\
\text { nascidos de mães adolescentes } \\
\text { com idade entre } 0 \text { e } 18 \text { meses; }\end{array}$ & $\begin{array}{l}\text { Crianças do grupo A (mães adolescentes), } \\
\text { apresentaram maior frequência de atraso, não } \\
\text { apresentado no grupo B. } \\
\text { Foi observada forte associação do } \\
\text { desempenho motor apenas com peso ao } \\
\text { nascer. Quando as associações foram } \\
\text { pesquisadas intra grupos, no grupo B não foi } \\
\text { encontrada associação significativa entre o } \\
\text { desempenho motor e as características } \\
\text { biológicas; no grupo A, foi encontrada } \\
\text { associação forte entre desempenho motor e } \\
\text { peso ao nascer (eta2 }=0,88 \text { ), sendo que os } \\
\text { bebês com maior peso ao nascer obtiveram } \\
\text { melhor desempenho motor. }\end{array}$ \\
\hline
\end{tabular}




\begin{tabular}{|c|c|c|c|}
\hline & & & $\begin{array}{l}\text { No presente estudo, observou-se diferença } \\
\text { significativa entre o desempenho dos bebês } \\
\text { filhos de adolescentes e os nascidos de mães } \\
\text { adultas, ressaltando a possibilidade de a idade } \\
\text { materna ser um fator de risco para atraso no } \\
\text { desenvolvimento }\end{array}$ \\
\hline Panceri, et al., 2012 & $\begin{array}{l}\text { Delineamento } \\
\text { quantitativo, } \\
\text { transversal, } \\
\text { comparativo e } \\
\text { de cunho } \\
\text { descritivo }\end{array}$ & $\begin{array}{l}\text { Foram avaliados } 12 \text { bebês de } 4 \text { a } \\
15 \text { meses de idade, divididos em } \\
\text { dois grupos pareados de acordo } \\
\text { com a idade corrigida e renda } \\
\text { familiar, tentando garantir, assim, } \\
\text { a equivalência quanto aos } \\
\text { aspectos socioeconômicos. O GH } \\
\text { foi composto por } 6 \text { bebês } \\
\text { internados pelo período mínimo } \\
\text { de } 30 \text { dias na Unidade de } \\
\text { Internação Pediátrica do HCPA; e } \\
\text { o GC - grupo controle, foi } \\
\text { composto por } 6 \text { bebês } \\
\text { provenientes de creches e escolas } \\
\text { de educação infantil, os quais não } \\
\text { tiveram internações hospitalares } \\
\text { anterioreS. }\end{array}$ & $\begin{array}{l}\text { Quanto ao desempenho motor de todos os } \\
\text { bebês participantes }(\mathrm{n}=12) \text {, verifica-se que } \\
50 \%(\mathrm{n}=6) \text { demonstraram desenvolvimento } \\
\text { motor atrasado, } 25 \%(\mathrm{n}=3) \text { desenvolvimento } \\
\text { motor com suspeita de atraso e } 25 \%(\mathrm{n}=3) \\
\text { desenvolvimento motor adequado. } \\
\text { Os resultados sugerem que a hospitalização } \\
\text { prolongada influência de forma negativa o } \\
\text { desenvolvimento motor dos bebês internados } \\
\text { no HCPA. }\end{array}$ \\
\hline $\begin{array}{l}\text { Bueno, Castro \& } \\
\text { Chiquetti, } 2014\end{array}$ & $\begin{array}{l}\text { Trata-se de um } \\
\text { estudo } \\
\text { descritivo com } \\
\text { abordagem } \\
\text { analítica e } \\
\text { quantitativa }\end{array}$ & $\begin{array}{l}21 \text { lactentes pré-termos. Os } \\
\text { critérios de inclusão foram, } \\
\text { crianças com idade corrigida de } \\
\text { três a } 18 \text { meses nascidas pré- } \\
\text { termo. Foram adotados como } \\
\text { critérios de exclusão crianças que } \\
\text { apresentavam histórico de } \\
\text { internações hospitalares } \\
\text { recorrentes, síndromes congênitas, } \\
\text { patologias neurológicas e/ ou } \\
\text { problemas ortopédicos }\end{array}$ & $\begin{array}{l}\text { Considerando a idade corrigida para cálculo } \\
\text { dos escores percentílicos da AIMS, dos } 21 \\
\text { lactentes avaliados, } 61,9 \% \text { apresentou } \\
\text { desenvolvimento motor alterado (suspeito e } \\
\text { atraso). } \\
\text { A maioria dos lactentes permaneceu sob os } \\
\text { cuidados da mãe, sendo que apenas } 9,52 \% \text { da } \\
\text { amostra frequentaram creche pelo menos por } \\
\text { seis meses, estando essas dentro dos lactentes } \\
\text { que obtiveram um desempenho superior na } \\
\text { escala do desenvolvimento da AIMS. }\end{array}$ \\
\hline Gerzson, et al; 2016. & $\begin{array}{l}\text { Experimental, } \\
\text { com abordagem } \\
\text { quantitativa. }\end{array}$ & $\begin{array}{l}\text { Os } 59 \text { bebês incluídos no estudo } \\
\text { foram distribuídos aleatoriamente } \\
\text { em três grupos: } 18 \text { bebês } \\
\text { atendidos três vezes por semana } \\
\text { (G3X); } 23 \text { bebês atendidos uma } \\
\text { vez por semana (G1X) e } 18 \text { bebês } \\
\text { do grupo controle (GC). }\end{array}$ & $\begin{array}{l}\text { Os resultados observados neste estudo } \\
\text { apontaram que } \\
\text { O grupo que recebeu intervenção motora três } \\
\text { vezes por semana obteve uma diferença entre } \\
\text { as pontuações do pré para o pós-intervenção } \\
\text { maior na postura prono, sentado e em pé. }\end{array}$ \\
\hline $\begin{array}{l}\text { Borba, Pereira \& } \\
\text { Valentini, } 2017\end{array}$ & $\begin{array}{l}\text { Estudo } \\
\text { descritivo, } \\
\text { comparativo e } \\
\text { associativo }\end{array}$ & $\begin{array}{l}40 \text { bebês de } 0 \text { a } 18 \text { meses } \\
20 \text { bebês de mães adolescentes e } \\
20 \text { bebês de mães adultas. }\end{array}$ & $\begin{array}{l}\text { No grupo de mães adolescentes, os modelos } \\
\text { de regressão apresentaram maior número de } \\
\text { fatores relacionados à predição do } \\
\text { desenvolvimento infantil, sendo que esses } \\
\text { fatores tiveram maior capacidade de predizer } \\
\text { o escore de variável desfecho. Principais } \\
\text { preditores: idade do pai, escolaridade dos } \\
\text { pais, pais morando juntos, mãe que não } \\
\text { trabalha fora de casa, características do lar em } \\
\text { termos de espaço e brinquedos, práticas } \\
\text { parentais e conhecimento dos pais sobre o } \\
\text { desenvolvimento infantil. }\end{array}$ \\
\hline Vargas, et al., 2018 & $\begin{array}{l}\text { O estudo foi do } \\
\text { tipo descritivo }\end{array}$ & $\begin{array}{l}25 \text { crianças nascidas na MBM e } \\
\text { encaminhadas ao } \\
\text { acompanhamento no follow-up, }\end{array}$ & $\begin{array}{l}\text { Foi observado que o grau de escolaridade } \\
\text { materna está diretamente relacionado ao DM }\end{array}$ \\
\hline
\end{tabular}




\begin{tabular}{|c|c|c|c|}
\hline & observacional & $\begin{array}{l}\text { de acordo com o método Canguru. } \\
\text { As crianças selecionadas para a } \\
\text { pesquisa preenchiam os seguintes } \\
\text { critérios: lactentes do } 1^{\circ} \text { ao } 4^{\circ} \\
\text { trimestre de vida (idade } \\
\text { corrigida), estabilidade clínica e } \\
\text { neurológica, além de viabilidade } \\
\text { para realização da avaliação } \\
\text { motora através da AIMS. } \\
\text { Todas as crianças foram } \\
\text { prematuras, cuja IG ao } \\
\text { nascimento variou entre } 25 \text { e } 36 \\
\text { semanas, com uma média de } 32 \\
\text { semanas. O peso ao nascimento } \\
\text { variou entre } 730 \mathrm{~g} \text { e } 2376 \text { g. } \\
\text { A idade mínima das genitoras foi } \\
\text { de } 18 \text { anos e a máxima de } 45 .\end{array}$ & $\begin{array}{l}\text { dos bebês avaliados. } \\
56 \% \text { apresentaram resultados compatíveis } \\
\text { com o esperado para idade e } 44 \% \\
\text { apresentaram DM atípico. }\end{array}$ \\
\hline Delgado, et al; 2020 & $\begin{array}{l}\text { Estudo } \\
\text { transversal e } \\
\text { descritivo }\end{array}$ & $\begin{array}{l}\text { Os dados foram coletados entre os } \\
\text { meses de julho e dezembro de } \\
2017, \text { sendo a amostra constituída } \\
\text { por pacientes hospitalizados na } \\
\text { unidade de internação pediátrica } \\
\text { do Hospital Materno Infantil } \\
\text { Presidente Vargas (HMIPV), em } \\
\text { Porto Alegre (RS). Foram } \\
\text { incluídas no estudo crianças entre } \\
\text { quatro e } 17 \text { meses, clinicamente } \\
\text { estáveis, sem suporte de oxigênio } \\
\text { e com alta. } \\
\text { Foram avaliadas } 110 \text { crianças, } \\
\text { com média de idade de } 8,95 \\
\text { meses (DP } \pm 4,315 \text { ), sendo } 61 \\
\text { meninos (55,5\%). Quanto à idade } \\
\text { gestacional, } 24 \text { eram prematuros } \\
\text { ( } 21,8 \% \text { ), com idade gestacional } \\
\text { inferior a } 37 \text { semanas, e } 89 \\
\text { ( } 80,9 \%) \text { tinham peso adequado ao } \\
\text { nascer. }\end{array}$ & $\begin{array}{l}\text { Foi possível observar que }(63,6 \%, n=70) \\
\text { encontra-se aquém do esperado. Das crianças } \\
\text { com suspeita de atraso e atraso no } \\
\text { desenvolvimento motor, } 70 \%(\mathrm{n}=49) \text { estavam } \\
\text { com as vacinas atrasadas, havendo associação } \\
\text { entre essas duas variáveis } \\
(\mathrm{p}=0,005) \text {. As crianças cujas famílias recebem } \\
\text { benefício socioeconômico também } \\
\text { apresentaram maior atraso no } \\
\text { desenvolvimento motor ( } \mathrm{p}=0,036) \text {. }\end{array}$ \\
\hline
\end{tabular}

Fonte: Dados da pesquisa.

\section{Discussão}

Por meio das análises dos resultados foi possível perceber que os artigos abordaram diretamente a avaliação das habilidades motoras grossas de bebês prematuros. No entanto, em todos os artigos analisados constatou-se que a AIMS é considerada um instrumento de avaliação confiável, para mensurar o desempenho motor de prematuros até os 18 meses de vida. Foi nesta perspectiva que emergiram 2 eixos temáticos para orientar essa discussão: As baixas condições socioeconômicas e atraso no desenvolvimento motor; e O tempo prolongado de internamento na UTIN e o desempenho motor.

\subsection{As baixas condições socioeconômicas e atraso no desenvolvimento motor}

De acordo com o Ministério da Saúde (2014), os primeiros anos de vida correspondem ao período de maior vulnerabilidade, o que coloca o recém-nascido em situação de maiores riscos, sociais, biológicos, socioeconômicos e 
culturais. Estudo realizado por Borba, Pereira \& Valentini (2017), afirma que a criança apresenta inúmeras conexões com o meio e que por este motivo o desenvolvimento infantil encontra-se correlacionado a diversos fatores. Para os autores, a gravidez precoce além de apresentar situações biológicas influentes, também existe a forte relação com os aspectos socioeconômicos nos quais as mães adolescentes estão inseridas, a situação de pobreza bem como contexto sociocultural ruim, e a falta de rede de apoio social tendem a influenciar negativamente sob as experiências vividas durante a maternidade na adolescência.

Neste estudo a forte relação entre desenvolvimento motor e situação socioeconômica foi percebida quando aplicado a escala de avaliação Alberta Motor Infant Scale (AIMS),instrumento que avalia a atividade motora ampla das habilidades apresentadas por neonatos até os 18 meses de vida, nas posições de prono, supino, sentado e em pé, sendo possível observar através da pontuação obtida e mostra percentílica como está o desenvolvimento da criança, podendo este último ser classificado em suspeito (percentil entre 5 e 25) atrasado (percentil <5) ou normal (percentil >25), os resultados encontrados no estudo sugerem que 35\% das crianças apresentaram desenvolvimento motor suspeito e $15 \%$ desenvolvimento motor atrasado; o que coloca uma mostra percentílica de 50\% das crianças com tendência a apresentar desenvolvimento motor atípico.

Ainda segundo estudo realizado por Borba, Pereira \& Valentini (2017), o próprio contexto familiar pode apresentar mudanças, uma vez que essas mães adolescentes começam a trabalhar muito mais cedo, ficando os filhos com as avós e não em creches, sendo estes fatores correlacionados ao desenvolvimento infantil. Este achado em discussão corrobora com os achados do estudo de Bueno, Castro \& Chiquetti (2014), no qual destacam o processo de desenvolvimento infantil como sendo multifatorial, afirmando que o contexto familiar (condições de saúde, educação, recursos sociais e práticas de cuidado) também deve ser analisado. Ademais, o estudo também destacou que um número expressivo de lactentes permaneceu sob os cuidados das mães e apenas uma pequena parte frequentou creches; estando estes últimos com as melhores respostas no processo de avaliação pela Alberta Infant Motor Scale (AIMS), 61,9\% das crianças obtiveram mostra percentílica caracterizando o desenvolvimento motor como (suspeito ou atrasado).

Bueno, Castro \& Chiquetti (2014), referem ainda que a condição socioeconômica familiar apresenta forte influência nos espaços físicos de desenvolvimento da criança, sendo que em cenários marcados pela pobreza a disponibilidade de brinquedo e materiais didáticos tendem a ser reduzida, e, portanto, as oportunidades de estímulos também, apresentando como consequência o atraso de desenvolvimento infantil.

O estudo anterior entra em conformidade ao que foi exposto por Vargas e colaboradores (2018), no qual os autores identificaram o fator socioeconômico como determinante para o atraso de desenvolvimento motor, sendo observado que os lactentes de mães que apresentavam escolaridade básica apresentaram $60 \%$ de risco para atraso de desenvolvimento atípico quando avaliados pela escala de Alberta Infant Motor scale (AIMS), em comparação aos lactentes de mães de ensino superior.

Vargas e colaboradores (2018), traz consigo questões como: desnutrição, assistência precária, desestruturação familiar, baixa renda familiar, menor experiência materna e tantos outros aspectos que tendem a influenciar no diagnóstico do desenvolvimento motor, uma vez que contribuem para um ambiente desfavorável ao desenvolvimento das crianças. Por fim, os achados do estudo de Delgado e colaboradores (2020) corrobora de uma forma geral com todos os outros estudos citados acima, ao afirmar que os aspectos de formação e funcionamento do cérebro sofrem interferência dos processos biológicos e psicossociais presentes na fase pré-natal e primeira infância, participando desta forma no processo de desenvolvimento das crianças e na trajetória do mesmo. 


\subsection{O tempo prolongado de internamento na UTIN e o desempenho motor}

Panceri e colaboradores (2012) relatam que é durante a primeira infância em que se observa o maior amadurecimento do SNC, é também nessa fase em que os maiores ganhos na área afetiva, social, motora e cognitiva acontecem. O recém-nascido apresenta sua capacidade de aprendizagem diretamente correlacionada a plasticidade neural, que é justamente a condição do sistema nervoso central (SNC) em adaptar-se a diferentes estímulos presentes no meio. Se tratando, portanto, da interação com o meio, os autores destacam a hospitalização como um processo no qual observa-se uma grande quantidade de eventos negativos ao desenvolvimento infantil; uma série de experiências desagradáveis como: estímulos táteis, sonoros, medo, ansiedade, sensação de abandono, que acabam negativamente influenciando no processo de desenvolvimento.

De acordo com os autores supracitados, o ambiente pode agir como facilitador ou reduzir as possibilidades de aprendizagem, quando em condições de hospitalização as possibilidades para o desenvolvimento tornam-se restritas, reduzindo o desempenho motor. Este fato pode ser observado no estudo quando $50 \%$ das crianças apresentaram desenvolvimento motor atrasado e $25 \%$ desenvolvimento motor suspeito.

O estudo de Giachetta e colaboradores (2010) corrobora com o estudo anterior na medida em que apontam que a sobrevivência dos recém-nascidos nas unidades de terapia intensiva neonatal acaba expondo recém-nascidos a situações de dor e estresse constantes, afirmando que o desenvolvimento neuropsicomotor está diretamente relacionado aos aspectos biológicos e ao tempo de internamento nas unidades de terapia intensiva neonatal.

Para os autores, o tempo de hospitalização deve ser considerado como fator de risco ao desenvolvimento, já que os recém-nascidos não conseguem absorver, organizar, e responder adequadamente a quantidade de estímulos negativos a que são expostos, apresentando como consequência comprometimento do desenvolvimento motor.

Em um estudo longitudinal realizado por Manacero \& Nunes (2021) sobre o comportamento do sono e do desenvolvimento motor em crianças prematuras com baixo peso a nascer, desde a infância até a pré-escola, os autores afirmam que o aumento das taxas de sobrevida de crianças prematuras relaciona-se com a melhoria do cuidado prestado ao recémnascido nas unidades de terapia intensiva, porém este fator não se apresenta ligado a redução das morbidades. Corroborando com o este estudo, Lawlor, et al., (2018) aponta a necessidade de programas de acompanhamentos e das equipes multiprofissionais, já que existe a necessidade de se intervir precocemente na identificação dos fatores de riscos, das alterações sensório motoras no qual os lactentes estão expostos pós alta da unidade de terapia intensiva neonatal, bem como no melhor tratamento ofertado visando os cuidados do recém-nascido.

\section{Conclusão}

Os resultados desse estudo evidenciaram que a Alberta Infant Motor Scale (AIMS) é considerado um instrumento de triagem do desenvolvimento motor grosso, (Balbi, Carvalhaes, \& Parada, 2016) confiável para ser aplicados em bebês prematuros, podendo ser aplicado de forma isolada ou associado com outros instrumentos de avaliação, sendo capaz de avaliar o desenvolvimento motor em relação aos marcos de desenvolvimento e transições de posturas.

Constatou-se também nos resultados do estudo que a baixa condição (Carvalho, Coelho \& Bacelar, 2016) socioeconômica das famílias de prematuros e o tempo de internamento (Bueno, Castro \& Chiquetti, 2014) prolongado nas Unidades de Terapia Intensiva Neonatal (UTIN) influenciou negativamente para o desempenho motor grosso dos bebês avaliados, assim como terapêutica pós alta da UTIN (Borba, Pereira \& Valentini, 2017; Ferreira, Alves, Guimarães, Menezes \& Magalhães, 2020). A cerca das considerações obtidas ao longo do estudo torna-se imprescindível a realização de pesquisas e 
aprofundamento da temática, devido a escassez destes na literatura científica, já que refletem a necessidade e importância dos instrumentos de triagem da avaliação do desenvolvimento motor infantil na assistência à saúde da criança.

\section{Referências}

Alves, R. \& Campos, D. (2011). Intervenção precoce em lactentes prematuros. Fisioterapia Brasil, 12, 374-375. DOI: https://doi.org/10.33233/fb.v12i5.942

Balbi, B., Carvalhaes, M. A., \& Parada, C. M. (2016). Tendência temporal do nasciemnto pré-termo e seus determinantes em uma década. Ciência \& Saúde Coletiva, 233-241. DOI: 10.1590/1413-81232015211.20512015

Borba, L., Pereira , K., \& Valentini, N. (2017). Motor and cognitive development predictors of adolescents and adults mothers. Journal of Physical Education. DOI: $10.4025 /$ jphyseduc.v28i1.2811

Bueno, E., Castro, A., \& Chiquetti, E. M. (2014). Influencia do Ambiente Domiciliar no Desenvolvimneto Motor de Lactentes Nascidos Pré-Termo. Rev Neurocienc, 45-52. DOI: 10.4181/RNC.2014.22.914.8p

Carvalho, S., Coelho, J. M., \& Bacelar, D. Â. (2016). Fatores maternos para o nascimento de recém-nascidos com baixo peso e premauros: estudo de casocontrole . Ciência \& Saúde, 76-82. DOI: 10.4181/RNC.2014.22.914.8p

Delgado, D., Michelon, R. C., Gerzson, L., De Almeida , C., \& Alexandre , M. (2020). Avaliação do desenvolvimento motor infantil e sua associação com a vulnerabilidade social. Fisioterapia e Pesquisa, 48-56. DOI: 10.1590/1809-2950/18047027012020

Ferreira, R. d., Alves , C. R., Guimarães , M. A., Menezes, K. K., \& Magalhães , L. d. (2020). Effects of early interventions focused on the family in the development of children born preterm and/or at social risk: a meta-analysis. Jornal de Pediatria, 20-38. https://doi.org/10.1016/j.jped.2019.05.002

Formiga, C. K., Cezar, M. E., \& Linhares , M. B. (2010). Longitudinal assessment of motor development and sitting skill in preterm infants . Fisioterapia e Pesquisa, 102-7. https://doi.org/10.1590/S1809-29502010000200002

Fuentefria , R., Silveira , R., \& Pracianoy, R. (2017). Motor development of preterm infants assessed by the Alberta Infant Motor Scale: systematic article. Jornal de Pediatria, 328-42. DOI: http://dx.doi.org/10.1016/j.jped.2017.03.003

Gerzson, L. R., Catarino, B. M., Azevedo, K., Demarco, P., Palma, M., \& Almeida, C. (2016). Frequência semanal de um programa de intervenção motora para bebês de berçário. Fisioterapia e Pesquisa , 178-84. DOI: 10.1590/1809-2950/14923223022016

Giachetta, L., Nicolau, C., Costa, A. P., \& Zuana, A. (2010). Influência do tempo de hospitalização sobre o desenvolvimento neuromotor. Fisioterapia e Pesquisa, 24-9. https://doi.org/10.1590/S1809-29502010000100005

Lawlor, G. C. O, et al. (2018). Caracterização de variáveis clínicas e do desenvolvimento motor de recém-nascidos prematuros. Rev. APS. 21 (2): 177 - 181. DOI: https://doi.org/10.34019/1809-8363.2018.v21.16060

Manacero, S \& Nunes, M. L. (2021). Estudo longitudinal do comportamento do sono e do desenvolvimento motor em crianças prematuras com baixo peso ao nascer, desde a infância até a pré-escola. Jornal de Pediatria. Volume 97, Edição 1, páginas 44-51. Doi: https://doi.org/10.1016/j.jped.2019.10.010

Mello, E., Gallo, S., Goulart, F. C., Herrero, D., \& Gallo, P. R. (2014). Motor development of brazilian breastfeeding infants in. Journal of Human Growth and Development, 163-167. http://pepsic.bvsalud.org/pdf/rbcdh/v24n2/pt_07.pdf

Panceri, C., Pereira, K., Valentini, N., \& Sikilero, R. H. (2012). A influência da hospitalização no desenvolvimento motor de bebês internados no Hospital de Clínicas de Porto Alegre. HCPA, 161-168. https://seer.ufrgs.br/hcpa/article/view/25819/19176

Pereira A. S. et al. (2018). Metodologia da pesquisa científica. $1^{\text {a }}$ edição. Biblioteca Central da UFSM. https://repositorio.ufsm.br/bitstream/handle/1/15824/Lic_Computacao_Metodologia-Pesquisa-Cientifica.pdf?sequence=1\&isAllowed=y

Ribeiro, A. S., Borges , M. B., \& Formiga , C. K. (2010). Desenvolvimento motor de prematuros participantes de uma programa de intervenção precoce . Fisioterapia Brasil, 271-276. DOI: https://doi.org/10.33233/fb.v11i4.1408

Sá, F. E., Nunes , N. P., Gondim, E. J., Almeida , A. K., Alencar, A. J., \& Cardoso, K. V. (2017). Intervenção parental melhora o desenvolvimento motor de lactentes de risco: série de casos. Fisioterapia Pesquisa, 15-21. DOI: 10.1590/1809-2950/15828624012017

Sampaio , T. F., Nogueira, K. P., Pontes , T. B., \& Toledo , A. M. (2015). Comportamneto motor de lactentes prematuros de baixo peso e muito baixo peso ao nascer. Fisioter Pesq, 253-60. DOI: 10.590/1809-2950/13533022032015

Sartori, N., Saccani , R., \& Valentini , N. (2010). Camparação do desenvolvimento motor de lactentes de mães adolescentes e adultas . Fisioterapia e Pequisa , 306-11. https://doi.org/10.1590/S1809-29502010000400004

Silva, C. A., Brusamarello, S., Cardoso, F. G., Adamczyk , N. F., \& Neto, F. R. (2011). Desenvolvimento de prematuros com baixo peso ao nascer nos primeiros dois anos de vida. Paulista de Pedriatria, 328-35. https://doi.org/10.1590/S0103-05822011000300004

Vanin, L. K., Zattib, H., Soncinib, T., Nunes, R. D., \& Siqueira, L. B. (2019). Fatores de risco materno-fetais associados a prematuridade tardia. Paulista de Pediatria, 38. https://doi.org/10.1590/1984-0462/2020/38/2018136

Vargas , M. C., Mendonça, A. S., Vieira , A. G., Lameira , A. B., \& Guinther , N. S. (2018). Avaliação de crianças atendidas em follow-up: perfil epidemiologico e motor. ConScientiae Saúde, 378-385. DOI: https://doi.org/10.5585/conssaude.v17n4.8532 